\title{
HEINRICH POMPEY
}

Zur Spiritualität des Helfens und des Helfers

Grundlagen des mittelalterlichen „Dienstes der Liebe“

Originalbeitrag erschienen in:

Giampeitro Dal Toso (Hrsg., u.a.): Nächstenliebe oder Gerechtigkeit? : zum Verhältnis von

Caritastheologie und Christlicher Sozialethik. Paderborn: Ferndinand Schöningh, 2014, Seite 41-63.

ISBN 978-3-506-77858-1 


\section{HEINRICH POMPEY}

\section{Zur Spiritualität des Helfens und des Helfers. Grundlagen des mittelalterlichen „Dienstes der Liebe“}

Kurz und prägnant hebt Benedikt XVI. 2012 in seinem Motu proprio über den „Dienst der Liebe“ mit Bezug auf seine erste Enzyklika Deus Caritas est (= DCE) hervor: „Der Dienst der Liebe ist ein konstitutives Element der kirchlichen Sendung und unverzichtbarer Ausdruck ihres eigenen Wesens (vgl. DCE, 25); alle Gläubigen haben das Recht und die Pflicht, sich persönlich dafür einzusetzen, das neue Gebot zu leben, das uns Christus hinterlassen hat (vgl. Joh 15,12), und dem modernen Menschen nicht nur materielle Hilfe zu bieten, sondern auch seelische Stärkung und Heilung (vgl. DCE, 28). " Ferner: „Gleichwohl ist es wichtig, sich zu vergegenwärtigen, dass ,die praktische Aktion zu wenig bleibt, wenn in ihr nicht die Liebe zum Menschen selbst spürbar wird, die sich von der Begegnung mit Christus nährt.' (DCE, 34)... Denn das karitative Wirken der Kirche muss sich auf allen Ebenen der Gefahr entziehen, einfach als eine Variante im allgemeinen Wohlfahrtswesen aufzugehen (vgl. DCE, 31)“. ${ }^{2}$

Neben der organisierten materiellen Hilfe - wie sie auch andere Dienstleister des Wohlfahrtswesens in den verschiedenen Ländern anbieten - ist das entscheidend Christliche am Dienst der Liebe die „seelische Stärkung und Heilung". Das bedeutet konkret: das materielle Helfen bedarf der spirituellen Ergänzung. Spiritualität verknüpft den Glaubensvollzug mit dem Lebensvollzug, d. h. verbindet existentiell Leib und Seele des einen und ganzen Menschen. ${ }^{3}$

Ebenso ereignet sich in der helfenden Beziehung, die eine existentielle Stärkung und Heilung intendiert, eine Lebens- und Leidensteilung ${ }^{4}$ zwischen Hilfebedürftigem und Helfer, die sich ,,von der Begegnung mit Christus nährt« (DCE, 34). Dadurch unterscheidet sich der Dienst der Liebe von anderen Praktiken öffentlicher, privater oder freier Anbieter medizinischer Dienstleistungen oder sozialer Beratungs-, Hilfs- und Begleitdienste.

Rein humanwissenschaftlich bedient sich der „Dienst der Liebe“ keiner anderen Medizin, benutzt keine anderen Psychologien, keine andere Pädagogiken, besitzt keine anderen physiologischen, pharmakologischen, operativen Voraussetzungen, etc. als andere Dienstleister auf dem Gebiet des individuellen sozialen Helfens und Heilens. Ihre „differentia specifica“ ergibt sich aus

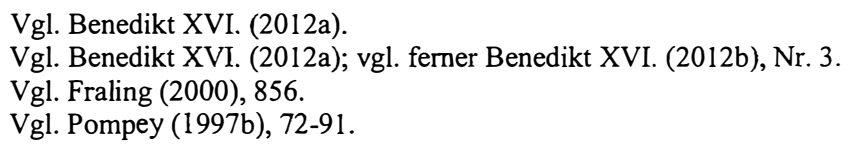


der Spiritualität ihrer helfenden Zuwendung. Außerdem ist sie bei der Anwendung pädagogischer, psychologischer oder physiologischer Hilfsmaßnahmen an die christliche Individualethik gebunden.

Die Spiritualität des Helfens stellt eine existentielle „Wirk“-lichkeit dar, die sich aus dem Sein Gottes und seiner Zuwendung zum Menschen ergibt. Sie ist vorrangig „theo-logisch“ geprägt, wie Benedikt XVI. im ersten Teil seiner Enzyklika Deus caritas est darlegt. Sie gründet in der erlösenden Beziehung Gottes zu den Menschen (vgl. DCE 1. Teil), die sich in Jesus Christus konkretisiert. Oder wie Papst Franziskus hervorhebt: „Wenn wir sagen, dass der Christ ein spiritueller Mensch ist, dann meinen wir genau das: der Christ ist ein Mensch, der Gott gemäß, dem Heiligen Geist gemäß denkt und handelt." "s

Spiritualität - als Teilhabe an der Mitleidenschaft Gottes in Jesus Christus (vgl. DCE, 10) - ereignet sich inkarnatorisch, d. h. durch bewusstes Hinabsteigen in die Leid-vollen und Schuld-beladenen Lebenswelten der Armut (Prototopos: Bethlehem) und der Migration (Prototopos: Herbergssuche und Flucht nach Ägypten) sowie in die Leidenswelt der psycho-sozialen Verlassenheit und Leidbetroffenheit (Prototopos: Getsemani, Gerichtshof mit Verspottung) wie in das physische Leid (Prototopos: Geißelung, Dornenkrönung, Kreuzweg, Kreuzigung). Der Helfende teilt so die Mit-leidenschaft Gottes in Jesus Christus, d. h. in seiner Inkarnation in die vier großen menschlichen Leidensbereiche.

Aus dem „Gott-ähnlich-Sein“ des Menschen mit dem trinitarischen Gott ergeben sich ferner gemäß der Enzyklika Deus caritas est zwei spirituell relevante Konkretionsweisen der caritativen Diakonie der Kirche: der „Dienst der Liebe“ und die „Gemeinschaft der Liebe“ (DCE, 19 Überschrift II. Teil). ${ }^{6}$ Christliche Caritas soll personal wie zugleich communial die trinitarische Caritas Gottes vergegenwärtigen. Darum stehen die helfende Beziehung zum Leidenden wie die caritative Kultivierung helfender Gemeinschaft (sei es z. B. durch Integration von Fremden und Ausgegrenzten in pfarrliche Gemeinschaften, durch treue, stabile und nachhaltige Betreuungskontakte, durch Pflege der Gastfreundschaft, d. h. Hospitalität für Leidende, Betagte und Arme ${ }^{7}$ etc.) im Vordergrund der caritativen Diakonie der Kirche; denn jedes Leid reißt persönliche wie zugleich soziale Wunden.

Um die berechtigte Sorge Benedikt XVI. aufzugreifen, nimmt dieser Beitrag exemplarisch die spirituellen Quellen des Dienstes der Liebe der Kirche vor der neuzeitlichen Organisation der Caritas in den Blick. Er fragt danach, ob zusätzlich zur Hl. Schrift oder aufbauend auf der Hl. Schrift spirituelle Traditionen die caritative Diakonie der Kirche noch heute inspirieren können

\footnotetext{
Franziskus (2013) 6.

Vgl. Pompey (2008), 387-419.

Vgl. das Lebenshaus im Malteserschloss zu Heitersheim oder die Lebensgemeinschaft der Trinitarier mit Migranten, Entlassenen etc. in Antequera Südspanien.
} 
und damit im Sinne der Anregungen von Benedikt XVI. das Profil des Helfens und der Helfer der Caritas der Kirche charakterisieren.

Der biblisch griechische Begriff pneumatikoj, d. h. „dem Geist gemäß“ ist das Ur-Wort für den später entstandenen lateinischen Begriff ,,spiritualitas“ ${ }^{\text {“ }}$ Gemeint ist, dem Hl. Geist gemäß zu handeln und zu leben. „pneumatikòoj“, d. h. „,dem Geist gemäß“ verstanden sich auch die ersten organisierten caritativen Einrichtungen und Dienste der Kirche seit dem Frühen Mittelalter. Die Pneumatologie wurde ihre praxisleitende Einrichtungsphilosophie.

Vorausgegangen war in der frühen süd-östlichen Kirche die helfende Begleitung von Menschen in Lebenskrisen - mit dem Ziel ,seelische Stärkung und Heilung“" zu vermitteln - durch die sog. Wüstenväter im 4./5. Jahrhundert. Analogie und Kompatibilität mit heutigen Praktiken des Helfens und Heilens sowie ihre Relevanz für soziales wie psychisches Helfen in der kirchlichen Caritas liegen in vielen Publikationen z. B. aus psycho-analytisch/psychiatrischer Sicht ${ }^{9}$ wie aus humanistisch-psychologisch-beraterischer Sicht ${ }^{10}$ vor. Die Darlegung dieser Form seelisch-existentieller Diakoniepraxis kann hier nicht ausgeführt werden, sondern bedarf einer eigenen Abhandlung.

Die Leitregeln für das materielle Helfen der frühen Kirche sind in der Didaskalia, der Didachae, der Apostolischen Konstitution und in den Schriften der Väter Justin (+ 165), Eusebius, Cyprian, Ambrosius hinsichtlich der Grenzen der materiellen Mittelbeschaffung, bezüglich der Voraussetzungen für den Empfang materieller Hilfen und der Konditionen auf der Seite des Helfers beschrieben. ${ }^{11}$ Diese materiellen Aspekte des Dienstes der Liebe werden in dem vorliegenden Beitrag nicht ausgeführt. Hier geht es nur um die Entfaltung der spirituellen Grundlagen des Dienstes der Liebe für Arme, Ausgegrenzte, Kranke etc. im europäischen Mittelalter.

\section{Das Leitbild des Hl. Geistes als „pater pauperum“}

Erste soziale Hilfezentren der Kirche im Frühen Mittelalter wurden in der Westkirche Diakoniae ${ }^{12}$ bzw. Armenhospitäler genannt. ${ }^{13}$ Sie waren soziale Service-Stationen und sind nicht mit Krankenhäusern unserer Zeit vergleichbar. Sie dienten nicht der Heilbehandlung, sondern der Armenpflege. Sie waren Häuser für Menschen, die Not litten, die keine Familie besaßen, keine

\footnotetext{
Vgl. Sudbrack (2000), 852.

Vgl. Hell $\left(2010^{3}\right)$.

Vgl. Bäumer, Plattig (2012).

Vgl. z. B. Didachae (2012); Apostolische Konstitution (1874), 2,4, 4,9 und 8,45; Ambrosius von Mailand, De officies ministrorum (1934-1938), 1. Buch, XXX, Nr.149.

12 Vgl. Pompey (2006), 109.

13 Vgl. Pompey (2000), 339-361. In der Ostkirche gründete Basilius d. Gr. sog. Städte der Barmherzigkeit, vgl. Valica (2008), 136.
} 
Bleibe hatten. Sie waren ein gastliches Haus, eine Herberge (hospitium, lat.) der christlichen Gemeinde bzw. einer christlichen Gemeinschaft (Orden, Bruderschaft etc.) für Menschen, die akute Hilfe - sei sie materieller, physischer oder sozialer Art - benötigten. Sie waren Notfallstationen für Arme, Heimatlose, Kranke, Flüchtlinge, Migranten und Pilger. In ihnen wirkten keine Ärzte sondern pflegerisch bzw. sozial tätige Helfer. Ärzte wurden nur zu Krankenbehandlungen konsultiert. ${ }^{14}$ Die Hospitäler waren Sozialküche, Erste-HilfeStation, Übernachtungsquartier für Arme und Wohnungslose, kurzfristiger Aufenthaltsort, Alten- und Pflegeheim, Unterkunft für verarmte Witwen und Waisen im Sinne von erweiterten sozialen Servicezentren wie sie in der Caritas der Universalkirche heute in vielen Ländern anzutreffen sind.

Eine der größten caritativen Einrichtungen des Frühmittelalters, die König Ina von Wessex (689-726) in Rom für englische Pilger in Not errichtet hatte, erneuerte Papst Innozenz III. (1160 - 1216) und weihte diese soziale Herberge (Hospiz) für Arme, Entrechtete, Wohnungslose, mittellose Kranke etc. dem Hl. Geist. Diese Einrichtung ist noch heute als Arciospedale di Santo Spirito in Sassia für Kranke existent. Gleichzeitig wurde die 1180 in Montpellier durch Guy de Montpellier gegründete Hospitalbruderschaft vom Hl. Geist mit den Diensten in diesem Hospital beauftragt und die Bruderschaft 1198 als Ordensgemeinschaft päpstlich anerkannt. Sicher geschah die Namensgebung nicht von ungefähr. Der Name sollte den Geist des Hauses (Leitungskultur) und das Hilfeverhalten (Hilfekultur) der Brüder bestimmen.

Die vom $\mathrm{Hl}$. Geist erbetene Spiritualität des stärkenden und heilenden Helfens wird im Mittelalter in Hymnen zum Hl. Geist verdichtet beschrieben. So z. B. durch Hrabanus Maurus (780-856) - Bischof von Mainz - , der den eindrucksvollen Hymnus zum Hl. Geist Veni creator Spiritus verfasste und damit die Synergie des Hl. Geistes mit dem Träger, dem Confirmatus des Hl. Geistes - sei er Helfer oder Hilfesuchender -, beschreibt.

14 Die Ausübung der Heilkunst medizinischer oder chirurgischer Art war Mönchen und Klerikern durch Päpste, Synoden und Konzilien im 12. Jahrhundert untersagt. Medizin und Chirurgie (Barbiere etc.) wurden als Dienstleistungen von Laien in den Städten ausgeübt. VgI. Pompey (1968), 23-26. 


\section{Veni creator Spiritus}

\begin{tabular}{|c|c|c|}
\hline $\begin{array}{l}\text { Hymnus nach Hrabanus } \\
\text { Maurus } \\
780-856\end{array}$ & $\begin{array}{l}\text { Deutsche Übersetzung } \\
\text { Wörtlich übersetzt } \\
\text { von Martin Bachmaier }\end{array}$ & $\begin{array}{l}\text { Eigenschaften des } \\
\text { Hl. Geistes als hel- } \\
\text { fende Aspekte in } \\
\text { heutige Terminolo- } \\
\text { gie übersetzt }{ }^{15}\end{array}$ \\
\hline $\begin{array}{l}\text { Veni creator Spiritus, } \\
\text { mentes tuorum visita, imple } \\
\text { superna gratia quae tu crea- } \\
\text { sti pectora. }\end{array}$ & $\begin{array}{l}\text { Komm, Schöpfer Geist, die Ge- } \\
\text { sinnungen der Deinen besuche; } \\
\text { erfülle mit oberer Gnade die } \\
\text { Herzen, die Du geschaffen hast! }\end{array}$ & $\begin{array}{l}{ }^{*} \text { Helfender Inspira- } \\
\text { tor } \\
{ }^{*} \text { Quelle neuer Le- } \\
\text { bensmotivationen }\end{array}$ \\
\hline $\begin{array}{l}\text { Qui Paracletus diceris, } \\
\text { donum Dei altissimi, fons } \\
\text { vivus, ignis, caritas et spiri- } \\
\text { talis unctio. }\end{array}$ & $\begin{array}{l}\text { Der Du der Beistand genannt } \\
\text { wirst, des höchsten Gottes Ge- } \\
\text { schenk, lebendige Quelle, Feu- } \\
\text { er, Liebe und geistliche Sal- } \\
\text { bung. }\end{array}$ & $\begin{array}{l}\text { *Lebens-Berater } \\
\text { *Beistand Gottes } \\
\text { *Spender von Le- } \\
\text { benskraft }\end{array}$ \\
\hline $\begin{array}{l}\text { Tu septiformis munere, di- } \\
\text { gitus paterne dexterae, tu } \\
\text { rite promissum Patris, ser- } \\
\text { mone ditans guttura. }\end{array}$ & $\begin{array}{l}\text { Du Siebengestaltiger im Amt, } \\
\text { Finger der väterlichen Rechten, } \\
\text { Du nach heiligem Brauch Ver- } \\
\text { sprochenes des Vaters, mit Re- } \\
\text { de bereichernd die Kehlen. }\end{array}$ & $\begin{array}{l}\text { *Verbalisierungs- } \\
\text { helfer }\end{array}$ \\
\hline $\begin{array}{l}\text { Accende lumen sensibus, } \\
\text { infunde amorem cordibus, } \\
\text { infirma nostri corporis, vir- } \\
\text { tute firmans perpeti. }\end{array}$ & $\begin{array}{l}\text { Zünd' an das Licht den Sinnen, } \\
\text { gieß' ein die Liebe den Herzen, } \\
\text { das Schwache unseres Leibes } \\
\text { stärkend durch ununterbrochene } \\
\text { Tugend! }\end{array}$ & $\begin{array}{l}\text { *Optimierer der } \\
\text { Selbstwahmehmung } \\
\text { durch Hilfen zur } \\
\text { Selbsterkenntnis, } \\
\text { verbunden mit wert- } \\
\text { schätzender Liebe, } \\
\text { *Bestärker von phy- } \\
\text { sischen und morali- } \\
\text { schen Lebensener- } \\
\text { gien }\end{array}$ \\
\hline $\begin{array}{l}\text { Hostem repellas longius, } \\
\text { pacemque dones protinus, } \\
\text { ductore sic te praevio vite- } \\
\text { mus omne noxium. }\end{array}$ & $\begin{array}{l}\text { Mögest den Feind weiter zu- } \\
\text { rückstoßen und Frieden sofort } \\
\text { schenken! Mit Dir so als vo- } \\
\text { rausgehendem Lotsen mögen }\end{array}$ & $\begin{array}{l}\text { *Auflöser belasten- } \\
\text { der Lebens- } \\
\text { realitäten, } \\
* \text { Vermittler von } \\
\end{array}$ \\
\hline
\end{tabular}

15 Es wäre lohnend, die Aussagen des Hymnus für die heutige Sozialarbeit zu operationalisieren, vergleichbar den Basisfaktoren helfender Beziehungen, vgl. Tscheulin $\left(2001^{2}\right)$, Tscheulin, Splitter (2001), 363-372. 


\begin{tabular}{|c|c|c|}
\hline & wir alles Schädliche meiden! & $\begin{array}{l}\text { Versöhnung } \\
\text { *Wegbegleiter } \\
\text { *Helfende Hand }\end{array}$ \\
\hline $\begin{array}{l}\text { Per te sciamus da Patrem, } \\
\text { noscamus atque Filium, } \\
\text { teque utriusque Spiritum } \\
\text { credamus omni tempore. } \\
\text { Amen. }\end{array}$ & $\begin{array}{l}\text { Gib, dass wir durch Dich den } \\
\text { Vater verstehen und auch den } \\
\text { Sohn erkennen und an Dich, } \\
\text { beider Geist, zu jeder Zeit glau- } \\
\text { ben! } \\
\text { Amen. }\end{array}$ & $\begin{array}{l}\text { *Quelle rechter Le- } \\
\text { benserkenntnisse, z. } \\
\text { B. Gottes Wollen, d. } \\
\text { h. seinen Willlen } \\
\text { zum Wohl des Men- } \\
\text { schen zu verstehen }\end{array}$ \\
\hline
\end{tabular}

Der Hymnus beschreibt wichtige vom Geist geschenkte Begabungen und Fähigkeiten, um Notleidenden zu helfen, aus einer existentiellen Krise - sei es bedrückende Armut, Vertreibung aus der Heimat, Vergewaltigung, physische Behinderung etc. - herauszufinden. Nur wenn dank der Gaben des Hl. Geistes eine existentielle Stärke und Heilung erfolgt, können Lebenskreativität und Kraftressourcen wieder frei gesetzt und der Lebensweg fortgesetzt werden. ${ }^{16}$

Vermutlich in Anlehnung an das römische Erzhospiz für Arme, Entrechtete, kranke Pilger wurden in den neu entstehenden Städten Europas im 11. und 12. Jahrhundert ebenfalls für fremde und familienlose Arme Bürgerspitäler zum $H l$. Geist errichtet - angefangen von Bordeaux am Atlantik bis Tallinn in Estland, von Messina auf Sizilien bis Edinburgh in Schottland. ${ }^{17}$ Die Weihe dieser Einrichtungen zum Hl. Geist sollte den praxisleitenden Geist des Hauses bestimmen. Man war sich bewusst, dass neben der materiellen Hilfe auch spirituelle Hilfen zur Stärkung und Heilung der physischen, psychischen und sozialen Wunden erforderlich sind, die man als Früchte und Gaben des Hl. Geistes verstand, um durch sie das Leben - insbesondere das Leid-volle - in Glaube, Hoffnung und Liebe zu bewältigen. ${ }^{18}$

Warum - lässt sich fragen - wurden die Hospitäler nicht Einrichtungen zum barmherzigen Samariter genannt, was von der Aufgabenstellung her doch nahe gelegen hätte, sondern Stätten des Hl. Geistes? Mit Benedikt XVI. kann man sagen: „Wie alle Gaben Gottes, so verweisen auch Glaube und Liebe auf das Wirken des einen Heiligen Geistes (vgl. 1 Kor 13) ... Indem er die Liebe in uns ausgießt, lässt uns der Heilige Geist an der besonderen Hingabe Christi teilhaben: an seiner Hingabe als Sohn gegenüber Gott dem Vater und als Bruder gegenüber allen Menschen (vgl. Röm 5,5) ..." ${ }^{“ 19}$ Der Hl. Geist ist Urgrund

16 Vgl. auch Haarländer (2006), 124-126.

17 Dank der lateinisch-christlichen Community Europas im Hochmittelalter war das Management dieser Einrichtungen in den Städten Europas verblüffend gleich organisiert.

18 Vgl. Hilberath (1995), 255.

19 Benedikt XVI. (2012b), Nr. 4. Ebenso wird im Blick auf die Dienste der Kirche in Lumen gentium (II. Vatikanum) gesagt: der Hl. Geist teilt ,unter den Gläubigen ... seine Gaben aus 
des Dienstes der Liebe. Die Früchte der Einwirkung des Hl. Geistes sind im Sinne von Paulus (Gal 5, 16-26): Liebe, Freude, Friede, Langmut, Freundlichkeit, Güte, Treue, Sanftmut und Selbstbeherrschung (Gal 5,22 f), die keine rein selbstgewirkten Dienstleistungen bzw. Zuwendungsqualitäten des Menschen sind. Darum stellt 1997 z. B. die „Gemeinsame Erklärung zur Rechtfertigungslehre“ fest: „Allein aus Gnade im Glauben an die Heilstat Christi, nicht aufgrund unseres Verdienstes werden wir von Gott angenommen und empfangen den $\mathrm{Hl}$. Geist, der unsere Herzen erneuert und uns befähigt und aufruft zu guten Werken. ${ }^{620}$ Dies ist ein gravierender Aspekt gegenüber einer reinen Werkgerechtigkeit, die jede sozial-helfende Tat als in sich christlich ausreichend deklariert, ein Verständnis, das in der organisierten Caritas der Kirche heute nicht selten anzutreffen ist.

Fast zeitgleich zur Entstehung der Bürgerhospitäler konkretisiert im Hochmittelalter Stephan Langton, Kardinalerzbischof von Canterbury (1150-1228), mit seinem bekannten Hymnus vom Hl. Geist, Veni Sancte Spiritu, die Geist gewirkte Praxis des Bestärkens und Heilens in den sozialen HospitalEinrichtungen der damaligen Zeit.

und verteilt ... auch besondere Gnaden, durch die er sie geeignet und bereit macht, verschiedene für die Erneuerung und den weiteren Aufbau der Kirche nützliche Werke und Dienste zu übernehmen“ (Vatikanum II., Lumen Gentium 12).

20 Gemeinsame Erklärung zur Rechtfertigungslehre, 1997 n. 15, vgl. dazu auch Benedikt XVI. (2012b) Nr. 3. 
Veni Sancte Spiritus

\begin{tabular}{|c|c|c|}
\hline $\begin{array}{l}\text { Stephan Langton } \\
\text { (1150-1228), Erz- } \\
\text { bischof von Canterbury } \\
\text { (Gotteslob Nr. 243) }\end{array}$ & $\begin{array}{l}\text { „Veni Sancte Spiritus“, } \\
\text { wörtlich übersetzt von } \\
\text { Martin Bachmaier }\end{array}$ & $\begin{array}{l}\text { Spirituell helfende Fähig- } \\
\text { keiten } \\
\text { (in heutiger Terminologie z. } \\
\text { B. analog den Basisfaktoren } \\
\text { helfender Beziehungen) } \\
\text { als Eigenschaften des Hl. } \\
\text { Geistes beschrieben }\end{array}$ \\
\hline $\begin{array}{l}\text { Veni Sancte Spiritus } \\
\text { et emitte caelitus } \\
\text { lucis tuae radium. }\end{array}$ & $\begin{array}{l}\text { Komm, heiliger Geist, } \\
\text { Und sende vom Himmel } \\
\text { her Deines Lichtes Strahl. }\end{array}$ & $\begin{array}{l}\text { *Erheller dunkler Lebens- } \\
\text { abgründe } \\
\text { *Erleuchter }\end{array}$ \\
\hline $\begin{array}{l}\text { Veni pater pauperum, } \\
\text { veni dator munerum, } \\
\text { veni lumen cordium. }\end{array}$ & $\begin{array}{l}\text { Komm, Vater der Armen, } \\
\text { Komm, Geber der Gaben, } \\
\text { Komm, Licht der Herzen. }\end{array}$ & $\begin{array}{l}\text { *Väterlicher Beschützer der } \\
\text { Armen, } \\
\text { *Gaben schenkender Helfer } \\
\text { *Helfer der Herzenser- } \\
\text { kenntnis }\end{array}$ \\
\hline $\begin{array}{l}\text { Consolator optime, } \\
\text { dulcis hospes animae, } \\
\text { dulce refrigerium. }\end{array}$ & $\begin{array}{l}\text { Bester Tröster, } \\
\text { Süßer Gast der Seele, } \\
\text { Süße Erfrischung. }\end{array}$ & $\begin{array}{l}\text { *Optimaler Berater } \\
\text { *Empathischer, erquicken- } \\
\text { der Gast der Seele } \\
\text { *Vermittler von Entspan- } \\
\text { nung } \\
\text { *Unterstützer von Ruhe } \\
\text { stiftender Gelassenheit }\end{array}$ \\
\hline $\begin{array}{l}\text { In labore requies, } \\
\text { in aestu temperies, } \\
\text { in fletu solatium. }\end{array}$ & $\begin{array}{l}\text { In der Mühe bist du Ruhe, } \\
\text { In der Hitze Mäßigung, } \\
\text { Im Weinen Trost. }\end{array}$ & $\begin{array}{l}\text { *Moderator in hitzigen Si- } \\
\text { tuationen } \\
\text { *Tröster }\end{array}$ \\
\hline $\begin{array}{l}\text { O lux beatissima, } \\
\text { reple cordis intima } \\
\text { tuorum fidelium. }\end{array}$ & $\begin{array}{l}\text { O seligstes Licht, } \\
\text { Erfülle das Herzensinnere } \\
\text { Deiner Gläubigen. }\end{array}$ & $\begin{array}{l}\text { *Vermittler optimaler Le- } \\
\text { benserkenntnisse, die die } \\
\text { Tiefe des empfindenden } \\
\text { Herzens berühren }\end{array}$ \\
\hline $\begin{array}{l}\text { Sine tuo numine } \\
\text { nihil est in homine, } \\
\text { nihil est innoxium. }\end{array}$ & $\begin{array}{l}\text { Ohne deinen Wink } \\
\text { Ist nichts im Menschen, } \\
\text { Ist nichts unschuldig. }\end{array}$ & $\begin{array}{l}\text { *Vermittler von Lebens- } \\
\text { chancen dank seiner Geist- } \\
\text { Inspirationen und der War- } \\
\text { nung vor Lebens-Schäden }\end{array}$ \\
\hline
\end{tabular}




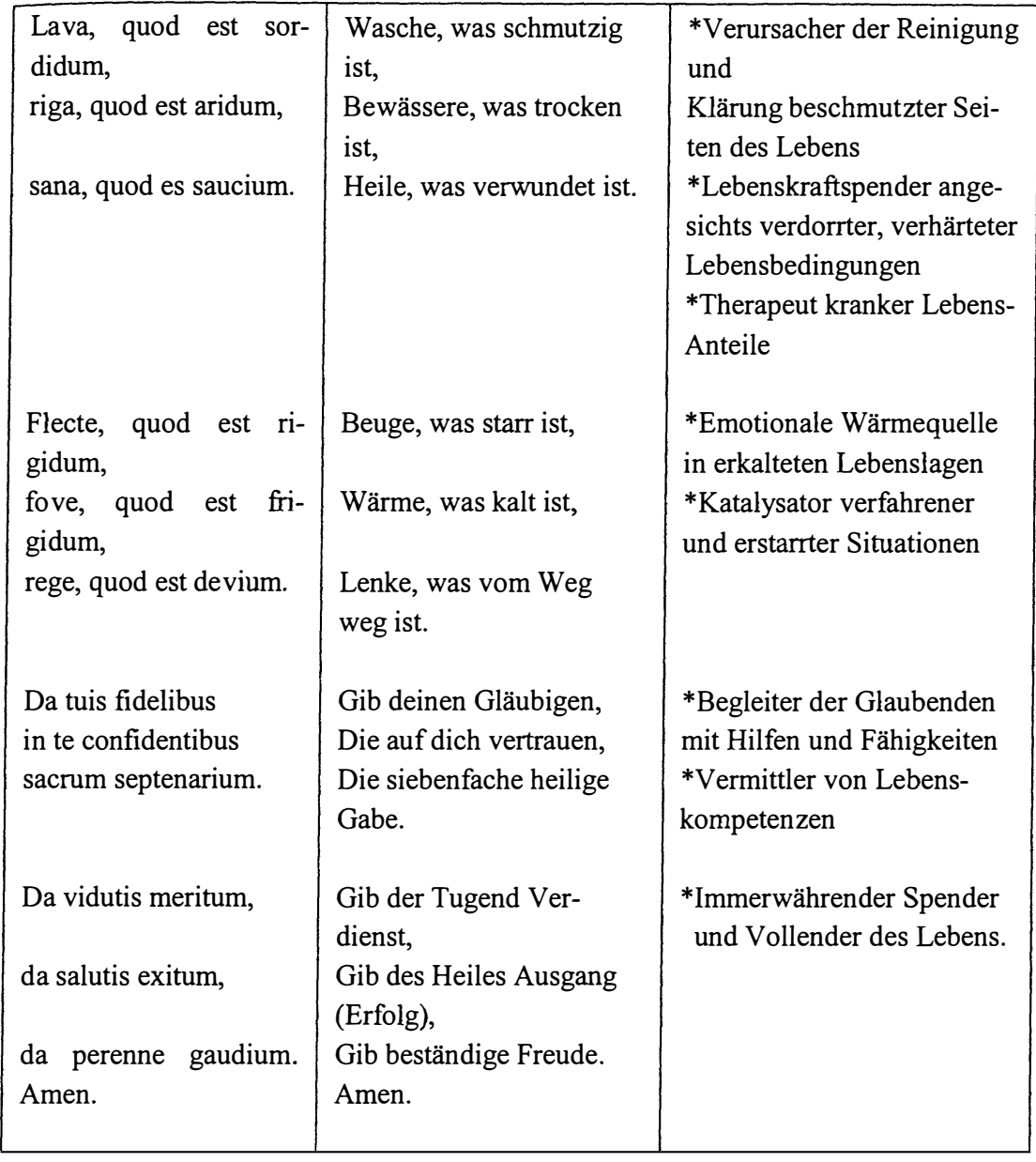

Der Geist Gottes - in Hymnen besungen - sollte den Dienst der Hospize bestimmen. Darum wurden die Armenhospize dem Hl. Geist geweiht.

Die helfenden Kompetenzen werden als Gaben des Hl. Geistes für den helfenden Christen bereits alttestamentlich bei Jes. 11,2 f. und vor allem von Paulus in seinem 1. Korintherbrief (1 Kor 12,4-11) beschrieben:

- Dem einen wird vom Geist die Gabe geschenkt, Lebensweisheit mitzuteilen,

- dem andern durch den gleichen Geist die Gabe, Erkenntnis zu vermitteln,

- dem dritten im gleichen Geist Glaubenskraft zu leben (somit anderen zu erschließen),

- einem andern die Gabe, Krankheiten zu heilen, 
- $\quad$ einem andern Wunderkräfte zu besitzen (d. h. effektive Lebensveränderungen herbeizuführen),

- einem andern prophetisches Reden (d. h. lebenskritische Verhaltensweisen klar zu benennen),

- einem andern die Fähigkeit, die Geister zu unterscheiden (d. h. aufzuzeigen, was hilfreich und was schädlich für die Lebensbewältigung ist),

- wieder einem andern verschiedene Arten von Zungenrede,

- $\quad$ einem andern schließlich die Gabe, sie zu deuten (d. h. Lebenssachverhalte richtig einzuschätzen, verstehend einzufühlen bzw. über die „Diakrisis“ [gr. = Unterscheidung] der „Logismoi poneroi“ [gr. der negativen bösen Gedanken] zu verfügen).

Wie Paulus herausstellt werden diese Gaben vom Geist geschenkt, damit sie anderen nützen, d. h. die Gaben sind nicht eigennützig zu verstehen. Es sind geschenkte Kompetenzen (Begabungen) der seelischen, geistigen, sozialen oder physischen Lebenshilfe. „Jedem aber wird die Offenbarung des Geistes geschenkt, damit sie anderen nützt" (1 Kor 12,4-11).

Die seit dem Mittelalter tradierten Gaben des Geistes lassen sich für das psychologisch-zwischenmenschliche Helfen wie folgt deuten ${ }^{21}$ :

- Die Gabe des Verstandes (d. h. Fähigkeit zur Rationalität), die die Lebenswahrheiten in ihrem wahren Sinn und ihrem inneren Ordnungsgefüge realistisch erfassen lässt.

- Die Gabe der Wissenschaft (d. h. Fach-Kenntnisse), die das gesammelte und gewachsene Lebenswissen als Lebenshilfe erschließen hilft.

- Die Gabe der Weisheit (d. h. Lebens-Klugheit und Lebenserfahrung), der die rechte Einschätzung der Welt im Lichte des Glaubens und der Vernunft gelingt.

21 Eine andere lebenspraktische Übersetzung findet sich im $\mathrm{LThK}^{2}$ (1995) 4.Bd., 479:

1. Die Gabe des Verstandes. Sie ist der Erleuchtung zugeordnet, die uns die Glaubenswahrheiten in ihrem wahren Sinn und ihrem inneren Ordnungsgefüge mit dem Blick auf unser Leben besser erfassen lässt.

2. Die Gabe der Wissenschaft, wo es um Glaubens- und Lebenswissen geht und die Fähigkeit, sie zu erschließen.

3. Die Gabe der Weisheit. Sie hilft uns zur rechten Einschätzung der Welt im Lichte des Glaubens und der Liebe.

4. Die Gabe des Rates, mit dem Gott selber uns zu einem sicheren praktischen Urteil führt, was wir im Sinne unserer Lebenslogik (Oikologik) auf unserem Heilsweg zu tun haben und was zu vermeiden ist. Sie unterstützt die Tugend der Klugheit.

5. Die Gabe der Frömmigkeit lässt uns Gott als unseren Vater verehren und macht uns frei von Sklaverei und Ängstlichkeit.

6. Die Gabe der Furcht schenkt uns Ehrfurcht vor Gott und damit Respekt vor jedem Menschen und vor dem Leben.

7. Die Gabe der Stärke gibt Kraft zum Vertrauen, das in Gott begründet ist. 
- Die Gabe des Rates (d. h. Beratungskompetenzen), die die Selbsterkenntnis und Selbstverantwortung fördert, z. B. was i. S. der Lebenslogik (Oikologik) und des Heilsweges zu tun oder zu vermeiden ist und was die Tugend der Klugheit fördert.

- Die Gabe der Frömmigkeit (d. h. Fähigkeit, Gottverbundenheit zu schenken ${ }^{22}$ ), die den Leid-beladenen in die eigene Gottesbeziehung (d. h. in das Gebet) einschließt und an der eigenen Glaubenserfahrung teilhaben lässt.

- Die Gabe der Furcht (d. h. die Gabe, den Respekt vor Gottes Willen zu wecken und die Selbstverantwortlichkeit des leidenden Menschen zu sensibilisieren), die Ehrfurcht vor Gott und inneren Respekt vor jedem Menschen und vor seinem Leben schenkt.

- Die Gabe der Stärke (d. h. die Fähigkeit, Mut und Demut beim helfenden Dienst zu praktizieren und von Gottes Heil-vollem Wirken überzeugt zu sein und so die Leidenden zu bestärken), die VertrauensKraft schenkt, die in Gott begründet ist. ${ }^{23}$

Um diese Gaben des HL. Geistes geschenkt zu bekommen, wurde seitens der Leidenden wie der Helfer in den Hospitalkirchen gebetet, insbesondere an den Pfingsttagen seit dem 12. Jahrhundert. ${ }^{24}$

In diesem Geist verbreiteten sich in den Städten des Mittelalters allgemeine soziale Anlaufstellen und Einrichtungen (Hospize). Bereits im Spät-Mittelalter spezialisierte sich das kirchlich-bürgerschaftliche Engagement in unterschiedliche pflegerische und soziale Dienste, für die die Gaben des Hl. Geistes ebenfalls praxisleitend blieben. Es entstanden Herbergen für bedürftige Pilger und Durchreisende, Valentinshäuser für Epileptiker, „Elendenherbergen“ für Arme, Waisenhäuser für Findelkinder, Aussätzigenhäuser, auch „Gutleutehäuser" genannt, Blatternhäuser für Syphilis-Kranke, etc. ${ }^{25}$

Bürgerschaftliches bzw. zivilgesellschaftliches soziales Engagement - getragen von einer Stadt oder einer Laienbruderschaft - bestimmte so neben dem sozialen Engagement der kontemplativen wie Bettel-Orden (Armenspeisungen an der Klosterpforte, Durchgangsherbergen, medizinische Notfallhilfen) und der Hospital- und Ritterorden (Heilig-Geist-Orden, Johanniter/Malteserorden, Deutschorden, Lazarusorden, u. a.) für Pilger und Migranten die caritative Diakonie im Mittelalter.

22 Ein zentrales Element caritativen Helfens nach Benedikt XVI.(2012b), Nr. 3.

23 Vgl. LThK ${ }^{2}$ (1995), 4. Bd., 479.

24 Vgl. die Sequenz: „Veni sancte spiritus“ in der Liturgie der Kirche seit dem 12. Jh.

25 Vgl. Pompey ( 2011), 41-68. 


\section{Das Leitbild der Barmherzigkeit}

Im 12. und 13. Jahrhundert herrschte eine große „Furcht ums Seelenheil“ sowie eine spirituelle „Endzeitstimmung“, die die von Christus in Gleichnissen und in der Rede vom Endgericht geforderten Werke der Barmherzigkeit in den Mittelpunkt der Metanoia stellten und so praktisch das caritative Helfen förderten..$^{26}$ Demgemäß wurden für das „obsequium pauperum“ die Aufrufe Jesu zur Barmherzigkeit als weitere Inspiration des „Dienstes der Liebe“ relevant. ${ }^{27}$

In den Gleichnissen Jesu zur Barmherzigkeit findet die Grundhaltung der Barmherzigkeit ihre konkrete Veranschaulichung (vgl. den barmherzigen Samariter, Lk 10,20-37; der barmherzige Vater, Lk 15,11-32). Dabei macht Jesus seinen Vater zum Maßstab für gelingende Menschlichkeit: „Seid barmherzig, wie Euer Vater im Himmel barmherzig ist" (Lk 6,36). Es geht also darum, durch Barmherzigkeit eine entsprechende lebensförderliche Beziehungskultur zu entfalten. Darum sagt Jesus: „Geht und tut das Gleiche“ (Lk 10,37).

Unbestritten ist das Gleichnis des barmherzigen Samariters eine Erzählung, mit der „Jesus die tiefe Liebe verständlich machen will, die Gott für jeden Menschen hegt, besonders wenn dieser krank ist und Schmerzen leidet", wie Benedikt XVI. hervorhebt. ${ }^{28}$ Der Samariter hilft dem sozial Armen, der niemanden hat, der allein und verlassen ist. Er nimmt eine Notversorgung vor (er ist kein Arzt) und transportiert ihn zum nächsten Hospiz (bindet ihn in ein Versorgungssystem ein). Er hilft dem zugleich materiell armen Straßenräuberopfer, indem er die Pflegekosten für ihn bezahlt und nach der Pflegezeit die Qualität der geleisteten seelischen und physischen Hilfe überprüft (Lk 15,1132).

Er ist b-armen-herzig, d. h. er schenkt dem Armen sein Herz. „Misericordia" heißt, denen es schlecht geht, d. h. den Armen, den Elenden, den Ausgegrenzten (miseri lat.) das Herz schenken (cor dare lat.). Dies zeichnet den Samariter aus und wird seinerseits vom Herbergsbesitzer erwartet. Denn Herbergen, respektive Gasthäuser für Notleidende dieser Art sollten nicht nur über ein organisatorisch qualifiziertes soziales und pflegerisches Management verfügen, wie es vermutlich auch der Wirt im Gleichnis praktiziert, sondern ebenfalls den rechten helfenden wie heilenden Geist atmen, d. h. eine entsprechende Unternehmens- und Hilfekultur besitzen, um der Unternehmensphilosophie (Unternehmensvision und Unternehmensmission einer Dienstleistungs-

26 Bereits Aurelius Augustinus (354 - 430) betont den Zusammenhang von Geben und Vergeben, wenn er herausstellt: „Es gibt zwei Arten, Gutes zu tun: Geben und Vergeben. Wegschenken, was man erworben hat und verzeihen, was man Böses erdulden musste." Auch wenn hier der Vergeber zugleich auch der Geber ist und nicht wie im Jüngsten Gericht Christus.

Vgl. Pompey (1983), 136-143; Glatzel N.; Pompey H. (Hg.) (1991), Pompey (1997a), 244 258.

28 Vgl. Benedikt XVI. (2013), 7. 
einrichtung - wie es in der Qualitätsmanagementtheorie heißt) Raum und Zeit zu eröffnen. Denn der Geist einer Einrichtung bestimmt die Qualität der Hilfe, vor allem wenn die Hilfe existentieller Natur sein soll. Der Geist einer Einrichtung muss - will sie ,seelische Stärkung und Heilung“ vermitteln - vom „pater pauperum“ geprägt sein. Diesen stärkenden und heilenden Geist der liebenden Zuwendung Gottes zu den Armen, den Jesus uns gesandt hat, beschreibt bzw. konkretisiert die Kirche in den genannten Hymnen als Gaben des Hl. Geistes.

Zivilgesellschaftliches Engagement aus diesem christlichen Geist der Barmherzigkeit wurde in den Bruderschaften als Hilfe für fremde, alte, behinderte, kranke Arme oder zum Besuch von Gefangenen, zum Freikauf von Sklaven, zur Bestattung von Verstorbenen (i. S. einer Fremdhilfegemeinschaft) betrieben. Die mittelalterlichen Zunftgemeinschaften praktizierten demgegenüber Werke der leiblichen Barmherzigkeit als Selbsthilfe- und Solidargemeinschaft für ihre Mitglieder (i. S. einer Selbsthilfegemeinschaft). Von der Gerichtsrede Jesu inspiriert, wandten sich sowohl die Mitglieder der Bruderschaften wie der Zunftgemeinschaften den Leidenden zu; denn nur der wird von Jesus beim Jüngsten Gericht als ihm ähnlich erkannt, der die Werke der Barmherzigkeit gelebt hat. Dementsprechend finden sich in fast allen Pilgerherbergen und Kommenden der Johanniter/Malteser Abbildungen des Jüngsten Gerichts. Zusätzlich zur Ausrichtung des Helfens an den Forderungen des Jüngsten Gerichtes wird beim Johanniterorden auch die Verbundenheit mit der Hl. Geist-Spiritualität z. B. beim Aufnahmeversprechen in den Hospitaldienst um 1198 zum Ausdruck gebracht. ${ }^{29}$

Nicht von ungefähr werden die Werke der leiblichen Barmherzigkeit im Mittelalter durch Werke der geistigen Barmherzigkeit ergänzt ${ }^{30}$, um die spirituellen und existentiellen Hilfen zur Leidbewältigung zu verstärken, z.T. ana$\log$ den Gaben des Heiligen Geistes. Die Werke der leiblichen Barmherzigkeit beschreiben in Anlehnung an die Gerichtsrede Jesu vorrangig verschiedene physische Dienstleistungen (visito, poto, cibo, redimo, tego, colligo, condo). Die geistigen Werke der Barmherzigkeit (consule, carpe [castiga $=$ zurechtweisen], doce, solare, remitte, fer, ora) verstärken sie spirituell. Sie werden jedoch im Kontext der Barmherzigkeitsforderung nicht als Gaben des hL. Geistes, sondern als Werke des Menschen vorgestellt; denn im Sinne der mittelalterlichen Scholastik sind die geistigen Werke der Barmherzigkeit Ausdruck der Tugend der Caritas (vgl. S. th. II-II, 32,2 ff.). Sie benennen auf ihre Weise spirituell relevante Hilfepraktiken.

29 Vgl. Klement (2010), 129.

30 Bereits Augustinus unterscheidet zwischen „beneficientia in corpus proximi“ und „beneficientia in animam proximi“, vgl. Bopp (1999), 1099. 
Die Werke der geistigen Barmherzigkeit ${ }^{31}$ besagen für das soziale Hilfeverhalten und für die Hilfe zur existentiellen Problembewältigung:

- consule: Guten Rat geben und andere an der eigenen Lebenserfahrung teilhabenlassen bzw. Zweifelnde beraten;

- carpe: Etwas klar und ehrlich sagen; Auf Verfehlungen aufmerksam machen, doch dabei die Wahrheit in Liebe sagen (Eph 4,15) und bei der geistlichen Hilfe selbst transparent, ehrlich und verantwortungsbewusst sein;

- doce: Unwissende lehren, so z. B. auf Hilfsansprüche aufmerksam machen, Hilfsmöglichkeiten wie Lebensformen aufzeigen und vermitteln;

- $\quad$ solare: Trauernde trösten, Verluste an Beziehungen und Sachen ertragen helfen, Verhärtungen auflösen, festgefahrene Situationen aufweichen;

- remitte: Helfen, Unrechtshandlungen und Beleidigungen zu verzeihen und seelische Verletzungen zu verarbeiten lernen, verhindern, dass sich Ärger, Wut, Vorurteile etc. festsetzen;

- fer: Zur-Last-Fallende geduldig unterstützen, seien es Angehörige, Kinder, Betagte, Behinderte, Freunde, Kollegen etc.;

- ora: Für Lebende und Verstorbene beten, d. h. Leidende in die eigene Beziehung mit Gott aufnehmen und so Gott danken und bitten für andere.

Die Werke der Barmherzigkeit beschreiben in ihrer Weise konkret physisch wie psychisch relevante, helfende Aspekte der liebevollen Zuwendung zu den Schwächsten und sind konkrete Formen des Dienstes der Liebe. Sie operationalisieren christlich-caritative Lebenskultur und damit Lebenskompetenzen.

\section{Das spirituelle Praxisleitbild der Tugenden}

Machen die Gaben des Hl. Geistes den Gott geschenkten Teil der helfenden Diakonie deutlich (= gifts of grace, abilities), so verweisen die Werke der Barmherzigkeit auf die aktiven Dienstleistungen der Helfer (= behavior). Doch darüber hinaus spielt die Tugendethik ${ }^{32}$ für die helfende soziale oder existentielle Beziehung in der mittelalterlichen Spiritualität der Orden und Ritterstände eine relevante Rolle, ebenso wie auch der Bürger im Hochmittelalter (attitudes/cognitive appraisals). Mit ihr werden somit neben den Begabungen und den konkreten Diensten auch die motivierenden Einstellungen/Haltungen/Bewertungen benannt.

\footnotetext{
${ }^{31}$ Vgl. Katholischer Erwachsenen-Katechismus (1995).

32 Vgl. Pinckaers (2004).
} 
Metaphorisch werden die Tugenden als Gegenkräfte zu den destruktiven Lastern in der sog. Psychomachia ${ }^{33}$ für die praktische Lebensgestaltung und Lebenskrisenbewältigung beschrieben. Die Laster gelten als Ursache psychosozialen Leids für die Betroffenen selbst wie für die Mitmenschen. Nur durch Förderung der Tugenden ist ihre schädliche Wirkung zu beheben. Bereits der im 4. Jahrhundert lebende spanische Dichter Prudentius (348 - 405) stellte in Form eines Epos den Kampf zwischen Tugenden und Lastern dar. ${ }^{34}$ Das Mittelalter bedient sich entsprechender Schemata, um das Zueinander von Tugenden (heute i. S. von Coping-Mechanismen zu verstehen) und Lastern (d. h. unsoziale bzw. selbst-zerstörerische Wünsche/Begierden/Schwächen) zu visualisieren, zu gliedern, zu systematisieren und für die spirituelle Förderung des Menschen zu operationalisieren. Sie wurden vertreten im frühen Mittelalter über Johannes Cassian (360-435) und Gregor d.Gr. ( 540-604) bis zum Hochmittelalter z. B. durch Hildegard von Bingen mit dem „Ordo virtutum“ oder Tenxwind von Andernach mit dem „Speculum virginum“.

\footnotetext{
${ }^{33}$ Ausgangspunkt für diese bildhafte Beschreibung der Psychomachia-Theorie bildet der Rat des Paulus in Eph. 6.11ff. zur Vorbereitung auf den geistlichen Kampf: „Legt die Waffenrüstung Gottes an, auf dass ihr standhalten könnt gegen die Ränke des Teufels...“

34 Vgl. Legner (1985), 69-80.
} 
Speculum virginum der Priorin Tenxwind v. Andernach um $1150^{35}$

\begin{tabular}{|c|c|}
\hline $\begin{array}{c}\text { Der alte Adam } \\
\text { nackt und verschlossen }\end{array}$ & $\begin{array}{c}\text { Der neue Adam } \\
\text { bekleidet, segnend und offen }\end{array}$ \\
\hline Fructus carnis & Fructus spem \\
\hline Luxuria & Caritas \\
\hline Ignorantia - Avaritia & Fides - Spes \\
\hline $\begin{array}{l}\text { Babylonia } \\
\text { sinistra }\end{array}$ & $\begin{array}{c}\text { Jerusalem } \\
\text { dextera }\end{array}$ \\
\hline Tristitia & Temperantia \\
\hline Imaginatio & Prudentia \\
\hline Superbia & Humilitas \\
\hline Verfehlung des Lebens & Findung der Lebens-Fülle \\
\hline $\begin{array}{l}\text { Männlich: Lebensfeindliche Begier- } \\
\text { den entspringen dem Kopf, dem } \\
\text { kognitiven Bereich }\end{array}$ & $\begin{array}{l}\text { Weiblich: Tugenden entspringen der } \\
\text { Brust, dem affektiven Bereich }\end{array}$ \\
\hline
\end{tabular}

Im Gegensatz zu den heute eher vorrangig ,ich-orientierten“ bzw. selbstverwirklichungs-orientierten psychologischen wie sozialpädagogischen Hilfen balanciert das Psychomachia-Konzept stärker das „bonum individuale“ und das „bonum commune“ aus. Die Tugenden galt es zur Überwindung selbstzerstörerischer und sozial destruktiver Kräfte prophylaktisch und therapeutisch helfend zu vermitteln. Da Glaube, Hoffnung und Liebe durch die Sieben Gaben des Hl. Geistes bestärkt werden ${ }^{36}$, erweist sich die Tugendethik wiederum als mit der Hl. Geist-Spiritualität vernetzt. Umgekehrt erwachsen aus Glaube, Hoffnung und Liebe gute Werke, die das Handeln Gottes an und im Menschen sichtbar machen. ${ }^{37}$

35 Transkription der farbigen Darstellung des Speculum virginum der Priorin Tenxwind v. Andernach um 1150, vgl. Legner (1985), 69-80.

36 Vgl. Hilberath, (1995), 253.

37 Vgl. Freitag (2001), 1097. 
So wurde von Thomas von Aquin (1225-1274) in seiner Almosenlehre ${ }^{38}$ insbesondere den Quaestiones zur Liebe, zur Barmherzigkeit etc. ${ }^{39}$ - bis zu Luis Vives (1492-1540), dem großen praxisorientierten Humanisten des ausklingenden Mittelalters, dank seiner Schrift: „De subventione pauperum“ der Zusammenhang zwischen der Tugend der Caritas und den Werken der leiblichen wie geistigen Barmherzigkeit differenziert untersucht und beschrieben. Wenn auch unterschiedlich, zeigen bzw. begründen beide, wie aus der Liebe die Barmherzigkeit erwächst. ${ }^{40}$ Die Bedeutung der Tugenden stellt auch Benedikt XVI. für den heutigen Dienst der Liebe als grundlegend in seiner Botschaft zur Fastenzeit $2013^{41}$ heraus. Ebenfalls verweist er auf die Tugenden als Grundhaltungen des Helfens in seiner Enzyklika „Deus caritas est“ und veranschaulicht sie im Schlusskapitel durch die Lebenszeugnisse heiliger Männer und Frauen der Kirche.

\section{Die praktische Relevanz des spirituell-existentiellen Helfens}

Inwiefern die beschriebenen Hilfefaktoren und das überlieferte Zusammenwirken der Begabungen des Geistes mit Werken der Barmherzigkeit und der Förderung der Tugenden seelisch existentiell hilfreich sind, sei abschließend kurz praktisch verdeutlicht, um die helfende Relevanz der Spiritualität einer Glaubens-, Hoffnungs- und Liebesdiakonie im Blick auf die von Benedikt XVI. für heute betonte „Bestärkung und Heilung“ zu verdeutlichen und $\mathrm{zu}$ operationalisieren ${ }^{42}$ :

Jedes schwere Leid - eine irreversible Behinderung, soziale Ausgrenzung als Migrant, Verlust eines geliebten Menschen, multi-morbides Altsein etc. schlägt nicht nur äußere Wunden sozialer, physischer oder psychischer Art, sondern verletzt stets auch innerlich und damit seelisch-existentiell, wie jeder Helfer aus seinen Hilfs-, Besuchs- und Begleitungsdiensten von schwer und irreversibel sozial, körperlich oder psychisch beschädigten Menschen weiß.

- Solche Menschen können oft nicht mehr glauben, dass in ihnen und in ihrem Leben noch irgendetwas Gutes zu finden ist. Der verbliebene Lebensrest ist für sie nur noch jämmerlich. Sie fühlen sich „Gott-

\footnotetext{
S.th. II/II q32 a2 vgl. Keck (2010), 63 - 93.

Vgl. Thomas von Aquin (1959), Quaestiones II. 23-33.

40 Keck (2010), 121.

41 Vgl. Benedikt XVI.(2012b), Nr. 4.

42 In der Renaissance wie in der Barockzeit wird Christus oft als Apotheker dargestellt, der z. B. die Tugenden als Medizin verabreicht.
} 
verlassen", also verlassen von Gott, $d$. h. von allem Guten verlassen ${ }^{43}$ und können nicht mehr an die Erlösungstat Jesu glauben.

- Solche Menschen können nicht mehr hoffen, dass ihnen die Zukunft gelingende Lebensmöglichkeiten schenken wird. Aus ihrer Sicht sind alle Lebenschancen dahin.

- Solche Menschen können sich und ihr Leben nicht mehr liebevoll annehmen. Am liebsten möchten sie ihr Leben wegwerfen, d. h. aus dem Leben scheiden.

Aus diesem Grund ist es für so Verzweifelte Lebens-Not-wendig, dass Mitmenschen im Geist der Enzyklika „Deus caritas est“ den Dienst des Glaubens, Hoffens und Liebens an ihnen leisten ${ }^{44}$ und - wie Benedikt XVI. in diesem Zusammenhang, sagt - dies mit Demut und Geduld tun (DCE, 39).

So geht es beim caritativen Dienst der heilenden und helfenden Nähe - insbesondere bei schwer Leidenden, z. B. bei einem irreversibel seelisch, sozial, materiell oder physisch angeschlagenen Menschen - konkret darum:

- den Glauben an das von Gott geschenkte Gutsein jeden Lebens (Gen $1,1-31)$ und damit den Glauben an das Gutsein des Menschen in das leidvolle, nicht mehr zu gelingen scheinende Leben eines solchen Menschen hineinzutragen, d. h. stellvertretend an das tiefe Gutsein dieses leidenden Menschen zu glauben, da er selbst angesichts seines Leidens, seiner Lebenskatastrophe nicht mehr an sich wie an sein Leben und damit an Gottes bleibende Präsens in seinem Leben glauben kann.

- Ferner geht es in der christlichen Lebensdiakonie darum, in hoffnungslosen Situationen beseelt bzw. geprägt zu bleiben von einer Hoffnung wider alle Hoffnung (Röm 4,18; 2 Kor 1,7; 1,10; 1 Kol 1,23). Im Blick auf einen Verzweifelten, einen seelisch Gebrochenen geht es um Hoffnung auf eine Lebensperspektive, die von kleinen, beglückenden Begegnungen der nächsten Stunde oder Tage bis zur Erfüllung des irdischen Lebens bei Gott reicht. Im Vertrauen auf Gott darf der Helfer diese Hoffnung dem Leidenden vermitteln und selbst von dieser Hoffnung leben.

- Zudem trägt christliche Lebensdiakonie Liebe in nicht mehr liebenswerte Lebenslagen oder in lieblose Leidensexistenzen hinein: Caritative Lebensdiakonie nimmt den Leidenden mit Menschenfreundlichkeit an, auch wenn sich der Leidende selbst in dieser Situation nicht akzeptieren kann. ${ }^{45}$

43 Das sind Situationen, denen wir in unseren eigenen Familien, in der Nachbarschaft, im Freundeskreis, in der Betriebsgemeinschaft, den pfarrgemeindlichen Alten- und Pflegeheimen oder im häuslichen Besuchsdienst etc. begegnen.

44 und zwar. als Christen untereinander wie auch gegenüber Nicht-Christen.

$45 \mathrm{Um}$ in dieser Weise zu helfen, sich mit seinem Leben trotz seines Kreuzes zu versöhnen geht es nicht darum, den Verlust an Leben bzw. Lebensmöglichkeiten in falscher Heroik oder As- 
Dies ist der Kern, die spezifische Herzenskompetenz des von Benedikt XVI. herausgestellten „Dienstes der Liebe“. Dies ist zugleich die grundlegende Weise, den Glauben im helfenden Tun zu bezeugen, die nur in der Verbundenheit mit Christus ihre volle Kraft entfalten kann. ${ }^{46}$

Denn stehen Menschen vor solchen unüberwindlichen, leidbeladenen Lebensgrenzen, dann sagen sie oft: „Wissen Sie, ich habe keine Kraft mehr, ich schaffe es nicht mehr....". Ferner sagen sie: „Was soll das alles noch, es hat doch keinen Sinn mehr..." Lebenskraft und Lebenssinnperspektiven brechen zusammen. Das Lebensurvertrauen ist tief angeschlagen. Ja, das Lebensurvertrauen kollabiert. Ein Lebensurvertrauens-Infarkt droht. Nur wenn die von unermesslichem Leid betroffenen Menschen durch eine Glaubens-, Hoffnungsund Liebesdiakonie - dank einzelner Begleiter oder einer bergenden Gemeinschaft - mitgetragen werden, finden sie wieder Lebenskraft und Lebenssinnperspektiven. Demut und Geduld wie Nähe und Treue - die soteriologischen Operationalisierungen von Raum und Zeit - sind dabei konstitutive theologische Bedingungen. In der Wüste - dem Symbolort für Nichtleben - hat sich Gott als der Nahe und Treue, d. h. als „Jahwe“ offenbart. Diese spirituelle Begleitung schließt eine gute Sach- und Fachhilfe nicht aus sondern ein, wie Benedikt XVI. hervorhebt. Glaube, Hoffnung und Liebe sind das Medikament zur Heilung des Lebensurvertrauens ${ }^{47}$, d. h. der inneren Heilung. Bei schweren Leid-Erfahrungen können die äußeren, die medizinischen oder materiell sozialen Hilfen nur nachhaltig wirken, wenn auch das Lebensurvertrauen geheilt wird..$^{48}$

zetik zu beschönigen, zu verharmlosen oder zu verherrlichen. Ein großes Problem stellt für viele Menschen sicher die fehlende Leidensfähigkeit dar, d. h. ein fehlendes Aushalten- und Annehmen-können. Dies sollte jeder Helfer und jede Helferin bei ihrem Dienst am Menschen realistischerweise einplanen.

46 Hieraus ergibt sich für alle Christen und insbesondere für die MitarbeiterInnen karitativer Dienste die Notwendigkeit des Glaubens, jener „Begegnung mit Gott in Christus [...], die in ihnen die Liebe weckt und ihnen das Herz für den Nächsten öffnet, so daß Nächstenliebe für sie nicht mehr ein sozusagen von außen auferlegtes Gebot ist, sondern Folge ihres Glaubens, der in der Liebe wirksam wird" (DCE 31 a). Der Christ ist ein Mensch, der von der Liebe Christi ergriffen ist, und deshalb ist er von dieser Liebe gedrängt - ,caritas Christi urget nos" (2 Kor 5,14) Vgl. Benedikt XVI. (2012.b), Nr. 1 u 3.

47 Es geht bei dieser Diakonie nicht primär darum, Glaube, Hoffnung und Liebe mit Worten einem Leidenden zuzusprechen, sondern in der Begleitung eines Leidenden von Glauben, Hoffnung und Liebe tief beseelt zu sein. Wenn die Grundhaltungen des Glaubens, Hoffens und Liebens nicht nur aus dem Kopf, sondern aus dem Herzen kommen, i. S. die Barmherzig-keit, schenken und eröffnen sie dem Leidenden neue Lebenskraft und Lebensinspiration. Psychologen nennen dies die Vermittlung von Lebensurvertrauen. Ohne Lebensurvertrauen kann ein Mensch sein Leben nicht meistern.

48 Glaube, Hoffnung und Liebe schenken helfende Kraft und Weisheit. Doch beim Dienst des Glaubens, Hoffens und Liebens zur Revitalisierung von Lebenskraft und Lebenssinn/Lebensperspektiven sind, so Benedikt XVI. Demut - d. h. Dien-Mut - und Geduld - d. h. Mit-erdulden im Sinne von Mit-tragen und Mitleiden - erforderlich (vgl. DCE 39). Denn wir kennen weder die Zeit noch die Stunde, zu der das Kreuz, das ein Mensch zu tragen hat, erleichtert wird, und wir wissen nicht, wann und wie ein Mensch von seinem Kreuz erlöst 
Somit gründet die Fähigkeit zum Dienst des stellvertretenden Glaubens, Hoffens und Liebens auf einer Begabung (Firmung) durch den Hl. Geist. Glaube, Hoffnung und Liebe kann man in sich selbst nicht produzieren. Sie ereignen sich als Gabe des Hl. Geistes, die der Begabte/Gefirmte geistlich zu kultivieren und in den Werken der Barmherzigkeit weiter zu schenken hat. ${ }^{49}$

\section{Zusammenfassung}

Der soziale wie existentielle „Dienst der Liebe“ sah und sieht sich 1. beschenkt durch den Hl. Geist. Seine Gaben bzw. Begabungen werden zum Wohl des Helfers wie seiner Mitmenschen geschenkt (abilities, gifts). Ferner wurde und wird 2. der Dienst der Liebe als erlernte und eingeübte Praxis der Nächstenliebe verstanden. Verschiedene physische, soziale und psychische Dienstleistungen werden in den Werken der Barmherzigkeit aufgezählt (behavior). Sodann beschrieb bzw. beschreibt 3. die Tugendethik die helfende und heilende Beziehungsqualität bzw. Beziehungskultur. Mit der Tugendethik werden zudem mit Blick auf den Klienten Aspekte des innerseelischen Kräftespiels (cognitive appraisals) zur innerpersonalen wie zwischenmenschlichen Überwindung lebensfeindlicher Einstellungen und Begehrlichkeiten (Laster) (attitudes) benannt. ${ }^{50}$

Begabungen, Fähigkeiten/Fertigkeiten und Grundhaltungen/Bewertungen sind auch für die heutige Praxis des „Dienstes der Liebe“ und der „Gemeinschaft der Liebe“ (DCE, 19) entscheidende Faktoren. Im Allgemeinen werden sie in der Hilfetradition der Kirche jedoch nicht systematisch i. S. einer zusammenhängenden Handlungstheorie der Diakonik vernetzt dargelegt, sondern als helfende Handlungsfaktoren aufgezählt und zusammengestellt präsentiert.

Die aufgezeigten überlieferten spirituellen Fundamente der Caritas: die Gaben des Hl. Geistes, die christologische Barmherzigkeit und die Lehre von den göttlich-menschlichen Tugenden geben der kirchlichen Caritasarbeit als Dienst der Liebe und als Gemeinschaft der Liebe ihre grundlegende und spezifische Prägung. Dadurch unterscheidet sie sich elementar von der fachlich gu-

wird. Die helfenden Grundhaltungen Demut und Geduld werden in der Begleitung eines Menschen in großer Not und schwerem Leid dadurch nochmals verstärkt, wenn wir diesen Dienst des Glaubens, Hoffens und Liebens zudem in Treue und Nähe leben und so stellvertretend Gottes Zusage vergegenwärtigen: Ich bin, der ich bin da, ich bin Euch nahe und treu, mag kommen was da kommen mag (Ex 3,14). So hat Gott sich angesichts des Leidens seines Volkes in Ägypten dem Moses in der Lebenswüste offenbart. Mit diesen Grundhaltungen begleitete die „Mutter der Barmherzigkeit“ Maria ihren Sohn durch das irdische Leben bis unter das Kreuz.

49 Vgl. Benedikt XVI. (2012b), Nr. 1 und 4.

so Die Verbundenheit dieser Elemente verdeutlicht Benedikt XVI. zum Abschluss seines Pontifikats in seiner Botschaft zur Fastenzeit 2013, vgl. Benedikt XVI. (2012b), Nr. 4. 
ten Sozialarbeit, die öffentliche oder private Träger solcher Dienste leisten. Durch die drei genannten spirituell relevanten Faktoren nimmt die Caritas der Kirche am caritativen Grundvollzug bzw. Grunddienst der Kirche teil und wird der Liturgie und Verkündigung wesensgleich, wie Benedikt XVI. herausstellt; denn der Dienst der Liebe ist ein konstitutives Element der kirchlichen Sendung und unverzichtbarer Ausdruck ihres eigenen Wesens (vgl. DCE, 25).

\section{Literaturverzeichnis}

Ambrosius von Mailand, De officiis ministrorum, Bibliothek der Kirchenväter (19341938): Eine Auswahl patristischer Werke in deutscher Übersetzung (BKV2). Fribourg.

Apostolische Konstitution (1874) in: Bibliothek der Kirchenväter, 1. Serie, Band 19. Kempten.

Bäumer, Regina; Plattig, Michael (2012): Aufmerksamkeit ist das natürliche Gebet der Seele - Geistliche Begleitung in der Zeit der Wüstenväter und der personzentrierte Ansatz nach Carl. R. Rogers - eine Seelenverwandtschaft?! Würzburg.

Benedikt XVI. (2006), Enzyklika: Deus caritas est. [= DCE] Verlautbarungen des Apostolischen Stuhls Nr. 171, DBK Bonn.

Benedikt XVI. (2012 a): Motu proprio über den Dienst der Liebe. Rom.

Benedikt XVI. (2012 b): Botschaft zur Fastenzeit 2013 - Der Glaube an die Liebe weckt Liebe - „Wir haben die Liebe erkannt, die Gott zu uns hat, und ihr geglaubt" Joh 4,16. Libreria Editrice Vaticana 2012.

Benedikt XVI. (2013): Geh und handle genauso. Botschaft zum XXI. Welttag der Kranken am 11. Februar 2013, in: Deutsche Tagespost v. 10.01.2013, 7.

Bopp, Karl (1999): Werke der Barmherzigkeit, II. Historisch, in: LThK ${ }^{3,}$ Bd. 10, 1099.

Didachae (2012), in: Online-Ausgabe der Bibliothek der Kirchenväter, Fribourg.

Fraling, Bernhard (2000): Spiritualität, IV. Systematisch-theologisch, in LThK ${ }^{3}$ Bd. 9, 856.

Franziskus (2013): Lernen mit den Augen Christi zu sehen - Das Wirken des Heiligen Geistes - Ansprache während der Generalaudienz am 8. Mai 2013 (Übersetzung Claudia Reimüller), in: Die Tagespost vom 11. Mai 2013, 6 .

Freitag, Josef (2001): Werke I, II. Systematisch-theologisch, in: LThK ${ }^{3}$ Bd. 10, 1097.

Gemeinsame Erklärung zur Rechtfertigungslehre. Augsburg 1997.

Glatzel, Norbert; Pompey, Heinrich. (Hg.) (1991): Barmherzigkeit oder Gerechtigkeit? Zum Spannungsfeld von christlicher Sozialarbeit und christlicher Soziallehre. Freiburg.

Haarländer, Stephanie (2006): Rabanus Maurus zum Kennenlernen - Ein Lesebuch mit einer Einführung in sein Leben und Werk (Publikationen Bistum Mainz). Mainz.

Hell, Daniel $\left(2013^{3}\right)$ : Die Sprache der Seele verstehen - Die Wüstenväter als Therapeuten. Freiburg $2010^{3}$.

Hilberath, Bernd Jochen (1995): Gaben des Heiligen Geistes, in: LThK ${ }^{3}$ Bd. 4, 255.

Katholischer Erwachsenen-Katechismus (1995). II. Bd. Leben aus dem Glauben. DBK (Hg.) Freiburg.

Keck, Andreas (2010): Das philosophische Motiv der Fürsorge im Wandel - Vom Almosen bei Thomas von Aquin zu Juan Luis Vives' De subventione pauperum. 
Würzburg 2010., Bd. 25, Studien zur Theologie und Praxis der Caritas und Sozialen Pastoral (Hg: Heinrich Pompey, Ursula Nothelle-Wildfeuer).

Klement, Katja (2010): Gottes Gastgeber - Die Ritter des Hospitals von Jerusalem Die vatikanische Handschrift Vat. Lat. 4852, Norderstedt.

Legner, Anton (Hrsg.) (1985): „Ornamenta ecclesiae“. Kunst und Künstler der Romanik - Katalog zur Ausstellung. Köln, Bd. 1.

Pinckaers, Servais (2004): Christus und das Glück - Grundriss der christlichen Ethik. Göttingen.

Pompey, Heinrich (1968): Die Bedeutung der Medizin für die kirchliche Seelsorge im Selbstverständnis der sogenannten Pastoralmedizin. Freiburg.

Ders. (1983): Die geistliche Barmherzigkeit in der gemeindlichen Krankenpflege, in: Lebendige Katechese 5 (1983), 136-143.

Ders. (1995): Christlicher Glaube und helfende Solidarität in der Diakoniegeschichte der Kirche, in: Kerber, W. (Hg.): Religion und prosoziales Verhalten, München, 75134.

Ders. (1997 a): Barmherzigkeit - Leitwort christlicher Diakonie, in: Die neue Ordnung 51 (1997), 244 - 258.

Ders. (1997 b): Caritas als lebensteilige, freie Vergeblichkeit - Caritas-philosophische Grundlagen des Helfens, in: Pompey, H. (Hg.): Caritas - Das menschliche Gesicht des Glaubens: Ökumenische und internationale Anstöße einer Diakonietheologie, Würzburg, 72-91.

Ders. (2000): Der Bischof als „Pater pauperum“ in der Diakoniegeschichte der Kirche. Ordo und Charisma in Verantwortung für die caritative Diakonie, in: Hillenbrand, K.; Niederschlag, H. (Hg.): Glaube und Gemeinschaft, Festschrift für Paul-Werner Scheele, Würzburg, 339-361.

Ders. (2006): Caritas professionell jedoch „häretisch“ - Liturgie feierlich jedoch folgenlos? Zur inneren Verbundenheit von Diakonie und Eucharistie sowie von Glauben und Liebe, in: Haslbeck, B.; Günther, J. (Hg.): Wer hilft, wird ein anderer - Zur Provokation christlichen Helfens - Festschrift für Isidor Baumgartner, Berlin, 99121.

Ders. (2008): Wie im Himmel, so auf Erden - Wenn Liebe göttlich wird .... - Kirche als Ikone der Dreifaltigkeit, in: Klasvogt, P.; Pompey, H. (Hg.): Liebe bewegt ... und verändert die Welt - Programmanzeige für eine Kirche, die liebt. Zur Enzyklika Deus caritas est Papst Benedikts XVI. Analysen - Perspektiven - Strategien, Paderborn, 387-419.

Ders. (2011): Das Engagement für Arme im ausklingenden Mittelalter und in der frühen Neuzeit: Katholische und reformatorische Prägungsfaktoren des neuen kommunalen und staatlichen Armenwesens am Beispiel der Stadt Straßburg mit Vergleichen zu Freiburg i. Br., in: Krimm, K.; Mussgnug, D.; Strohm, Th. (Hg.): Armut und Fürsorge in der frühen Neuzeit (Oberrheinische Studien Bd. 29) Ostfildern, 41-68.

Sudbrack, Josef (2000): Spiritualität, I. Begriff, in: LThK Bd. 9, 852.

Thomas v Aquin, Summa Theologica - Die deutsche Thomas-Ausgabe (1959), Bd. 17A Die Liebe, Heidelberg, Graz, Wien, Köln.

Tscheulin, Dieter $\left(2001^{2}\right)$ : Wirkfaktoren psychotherapeutischer Intervention. Göttingen (Unveränderter Neudruck 2001).

Tscheulin, Dieter; Splitter, Sabine (2001): Kurzbericht über ein Inventar für Beziehungsverhalten (IBV), in: Langer, I. (Hg.): Menschlichkeit und Wissenschaft. Festschrift zum 80. Geburtstag von Reinhard Tausch. Köln, 363-372. 
Valica, Mihai (2008): Eine heutige Philanthropiewissenschaft und Diakonietheologie im Kontext der Orthodoxen Lehre und der Tradition der Rumänisch-Orthodoxen Kirche. Bucuresti. 